NBI-HE-96-23

SU-4240-637

hep-lat/9606012

June 96

\title{
Geometrical Interpretation of the KPZ Exponents
}

\author{
J. Ambjørn and K. N. Anagnostopoulos \\ The Niels Bohr Institute \\ Blegdamsvej 17, DK-2100 Copenhagen Ø, Denmark \\ U. Magnea \\ Nordita, \\ Blegdamsvej 17, DK-2100 Copenhagen Ø, Denmark \\ and \\ G. Thorleifsson \\ Physics Department, Syracuse University, \\ Syracuse, NY 13244, USA
}

\begin{abstract}
We provide evidence that the KPZ exponents in two-dimensional quantum gravity can be interpreted as scaling exponents of correlation functions which are functions of the invariant geodesic distance between the fields.
\end{abstract}




\section{Introduction}

The calculation of the dressed scaling exponents is a milestone in the theory of two-dimensional quantum gravity [1, 2]. Strictly speaking, however, the derivation uses only finite size scaling arguments and involves only matter field correlators integrated over all space-time. The result can be formulated as follows: if we are given a conformal field theory where a scalar operator $\phi$ has scaling dimension $\Delta_{0}$ we know that the integrated correlator will scale with the volume $V$ as follows

$$
\int_{V} d^{2} x \int_{V} d^{2} y\langle\phi(x) \phi(y)\rangle \sim V^{2-2 \Delta_{0}}
$$

If the conformal field theory is coupled to quantum gravity the corresponding expectation value now includes the average over equivalence classes of metrics with $\int d^{2} x \sqrt{g(x)}=V$ and Eq. (代) is replaced by

$$
\left\langle\int_{V} d^{2} x \sqrt{g(x)} \int_{V} d^{2} y \sqrt{g(y)} \phi(x) \phi(y)\right\rangle \sim V^{2-2 \Delta}
$$

where the dressed scaling exponent $\Delta$ for a conformal field theory with central charge $c$ is related to the scaling exponent $\Delta_{0}$ in flat space by

$$
\Delta / 2=\frac{\sqrt{1-c+24 \Delta_{0} / 2}-\sqrt{1-c}}{\sqrt{25-c}-\sqrt{1-c}} .
$$

While these results are beautiful, one key ingredient is missing compared to the theory in flat space: the concept of a correlation length which diverges at the critical point. In fact, it has oocasionally been argued that there exists no such concept in quantum gravity since we integrate over all metrics. As we discuss below, however, a proper definition of invariant correlation length exists, but even with this definition at hand there are situations where the correlation length is not divergent in quantum gravity, although it diverges for the same matter system in flat space. For many Ising spins coupled to quantum gravity there exist convincing arguments which show that the spin-spin correlation length does not diverge at the critical point even if the transition is not first order [3].

The task of this article is to provide evidence that there exists a divergent correlation length associated to correlation functions of matter fields in two-dimensional quantum gravity coupled to matter with central charge in the interval $0<c<1$. First a notation of reparametrization invariant distance is needed in order that one can attribute a physical meaning to a divergent correlation length. One trivial suggestion is the following: for a fixed metric the concept of geodesic distance serves well as the definition of invariant distance. We can transport this notation to quantum gravity provided we perform the quantum average over metrics where the two marked points are precisely separated by a geodesic distance $R$. In this way a possible definition of a two-point correlator of $\phi(x)$ could be

$$
G_{\phi}(R ; \Lambda)=\int \mathcal{D}[g] \mathcal{D} \varphi \mathrm{e}^{-S_{G}-S_{M}} \int d^{2} x d^{2} y \sqrt{g(x) g(y)} \phi(x) \phi(y) \delta\left(D_{g}(x, y)-R\right) .
$$


In Eq. (1) $D_{g}(x, y)$ denotes the geodesic distance between $x$ and $y$ with respect to the given metric $g$. The integration $\mathcal{D}[g]$ is intended to be performed over equivalence classes of metrics, while $\mathcal{D} \varphi$ signifies the integration over the matter fields which are coupled to quantum gravity via the action $S_{M}(\varphi)$. $\Lambda$ is the cosmological constant.

While this definition is straightforward it has not been easy to use in a continuum context, i.e. in Liouville theory. An inconclusive attempt was pioneered by F. David [4], but the $\delta$-function constraint made it difficult to perform any detailed calculations. In the context of two-dimensional gravity and non-critical string theory, regularized by the use of dynamical triangulations [5 8, it is possible to implement the $\delta$-function constraint in Eq. (4). This was done in [9], using the so called transfer matrix formalism of dynamical triangulated surfaces [10 12] (see also [13, 14] for continuum transcriptions of this formalism). In [9] the discretized version of Eq. (4) was calculated in the case $\phi=1$ and in the scaling limit the following result was obtained:

$$
G_{1}(R ; \Lambda)=\Lambda^{4 / 3} \frac{\cosh \Lambda^{\frac{1}{4}} R}{\sinh ^{3} \Lambda^{\frac{1}{4}} R},
$$

In 15] this result was generalized to other scaling operators in pure two-dimensional quantum gravity and the concept of an operator product expansion was developed, the distance of separation between the various operators being geodesic in the same way as in Eq. (4).

As shown in [9, 16] an object like $G_{1}(R ; \Lambda)$ is a good probe of the fractal structure of space-time which is determined by the exponential decay of $G_{1}(R ; \Lambda)$. We expect

$$
G_{1}(R ; \Lambda) \sim e^{-c \Lambda^{1 / d_{H}} R},
$$

where $d_{H}$ is the Hausdorff dimension of our universes in the following sense: consider the ensemble of universes where two marked points are separated a geodesic distance $R$. We define $d_{H}$ by

$$
\langle V\rangle_{R} \sim R^{d_{H}}
$$

provided $R$ is not much larger than $1 / \Lambda^{1 / d_{H}}$. We conclude from Eq. (5) that the fractal dimension of space-time in two-dimensional quantum gravity is 4 , as first proved by different means in [10].

By a Laplace transformation it is possible to obtain the correlation functions for universes of fixed volume $V$ :

$$
G_{\phi}(R ; \Lambda)=\int_{0}^{\infty} d V \mathrm{e}^{-\Lambda V} G_{\phi}(R ; V) .
$$

For $\phi=1$ the correlation function $G_{1}(R ; V)$ is related to the average "area" of spherical shells $S_{1}(R ; V)$ of geodesic radius $R$ by

$$
S_{1}(R ; V)=\frac{1}{V} \frac{G_{1}(R ; V)}{Z(V)},
$$

where $Z(V)$ denotes the partition function of fixed volume, i.e.

$$
Z(V)=\int \mathcal{D}[g] \mathcal{D} \varphi \mathrm{e}^{-S_{G}-S_{M}} \delta\left(\int d^{2} x \sqrt{g(x)}-V\right) .
$$


An alternative and very natural definition of Hausdorff dimension is

$$
S_{1}(R ; V) \sim R^{d_{h}-1} \quad \text { for } \quad R \ll V^{1 / d_{h}} .
$$

By an inverse Laplace transformation of Eq. (5) it follows that $d_{h}=d_{H}=4$. However, in the case of matter fields coupled to gravity there exists no proof that $d_{H}=d_{h}$. If $d_{H}=d_{h}$ it is natural to introduce the finite size scaling variable

$$
x=\frac{R}{V^{1 / d_{h}}} .
$$

From Eqs. (11) and (6) we expect for $S_{1}(R ; V)$ a finite size scaling relation of the form

$$
S_{1}(R ; V) \sim V^{1-1 / d_{h}} F(x),
$$

where $F(x)$ has the following properties:

$$
F(x) \sim x^{d_{h}-1} \quad \text { for } \quad x \ll 1, \quad F(x) \rightarrow 0 \quad \text { for } \quad x \gg 1 .
$$

These relations are of course satisfied with $d_{h}=4$ in pure gravity 9, 18 and numerical simulations indicate that it is also true for $0<c<1$ [17, 18]. The finite size scaling arguments can also be applied to the more general correlators

$$
S_{\phi}(R ; V) \equiv \frac{G_{\phi}(R ; V)}{V Z(V)} .
$$

From Eq. (3) we have for a field with scaling exponents $\Delta_{0}$ in flat space and $\Delta$ after coupling to quantum gravity [17, 18]:

$$
\int_{0}^{\infty} d R S_{\phi}(R ; V) \sim V^{1-\Delta}
$$

From this scaling and the definition of $d_{h}$ it is tempting to conjecture the following scaling behavior:

$$
S_{\phi}(R ; V) \sim \frac{R^{d_{h}-1}}{R^{d_{h} \Delta}} f(x)=V^{1-\Delta-1 / d_{h}} F_{\phi}(x)
$$

where $f(0)>0$ and $F(x) \sim x^{d_{h}(1-\Delta)-1}$ for small $x$. This formula generalizes Eqs. (11)-(14) to the case where matter is coupled to quantum gravity. The corresponding formula in flat two-dimensional space reads:

$$
S_{\phi}^{(0)}(R ; V) \sim \frac{R}{R^{2 \Delta_{0}}} f(R / \sqrt{V}) .
$$

In this article we report on extensive computer simulations where we tried to verify Eq. (17). The finite size aspect of the formula, i.e. the volume dependence, has already been checked in 17]. However, until now no reliable results exist for the correlator itself, i.e. for the $R$ dependence. As explained above this $R$ dependence, i.e. the infinite correlation length, is the crucial feature of conformal field theory and we provide evidence that it exists also for two-dimensional quantum gravity coupled to a conformal field theory if we use the geodesic distance as a measure of length. 


\section{Numerical setup and results}

The numerical simulations are performed as follows: As discretization we use dynamical triangulations. In this formalism surfaces with spherical topology are constructed from equilateral triangles and we allow self energy and tadpole graphs to form in the dual $\varphi^{3}$ graph of the triangulation. To each vertex is associated a spin, either Ising or three-states Potts spin, depending on the model. A given spin interacts with the spins on the neighboring vertices. The Monte Carlo updating of the triangulations is performed by the so-called flip algorithm and the spins are updated by standard cluster algorithms. The flips are organized in "sweeps" which consist of approximately $N_{L}$ accepted flips where $N_{L}$ is the number of links of the triangulated surface. After a sweep we update the spin system. All this is by now standard and we refer to [19 22] for details about the actions or Monte Carlo procedures.

The results presented in this paper cover system sizes from 16000 to 128000 triangles and the number of sweeps is 1.7-5.0 $\times 10^{6}$. For the Ising and three-states Potts model on dynamically triangulated surfaces the critical temperatures $\beta_{c}$ are known 223, 24]. We use these values in the simulations.

Geodesic distances on the triangulations are defined either as the shortest link distance between two vertices or the shortest path through neighboring triangles. While these two distances can vary a lot for individually chosen points on a given triangulation, they are proportional when the average over the ensemble of triangulations is taken. We will report here the results obtained by the use of link distances. Our discretized length $r$ will be the number of links. Our discretized volume (or area) will be the number $N_{T}$ of triangles used in the computer simulations and our discretized scaling variable will be

$$
x \equiv \frac{r}{N^{1 / d_{h}}},
$$

where $N$ is the number of vertices on the surface. We denote the discretized distributions corresponding to $S_{1}(R ; V)$ and $S_{\phi}(R ; V)$ by

$$
\begin{aligned}
& n_{1}(r ; N)=\left\langle\sum_{j} \delta\left(D_{i j}-r\right)\right\rangle, \\
& n_{\phi}(r ; N)=\left\langle\sum_{j} \sigma_{i} \sigma_{j} \delta\left(D_{i j}-r\right)\right\rangle,
\end{aligned}
$$

where the indices $i$ and $j$ label vertices: $i$ is a random fixed vertex for each measurement and $j$ runs over all vertices of the given configuration. $D_{i j}$ is the link distance between the vertices labelled by $i$ and $j$.

Our first task is to determine $d_{h}$. If the scaling hypothesis is correct, both the distributions $n_{1}(r ; N)$ and $n_{\phi}(r ; N)$ will depend only on the scaling variable $x$ given by Eq. (19), except for the overall scaling factors $N^{1-1 / d_{h}}$ and $N^{1-\Delta-1 / d_{h}}$ respectively. Hence, we have to determine the best value of $d_{h}$ such that

$$
n_{1}(r ; N)=N^{1-1 / d_{h}} F(x), \quad n_{\phi}(r ; N)=N^{1-\Delta-1 / d_{h}} F_{\phi}(x)
$$

for all values of $r$ and $N$. This has already been done for the Ising model and the three-states Potts model for the $n_{1}(r ; N)$ correlator in [17, 18] and for $n_{\phi}(r ; N)$ 
in [17]. Our data is in very good agreement with the results obtained in [17, 18], namely $d_{h}=4$ for both the Ising model and the three-states Potts model coupled to gravity. Details of these measurements will be published elsewhere [25].
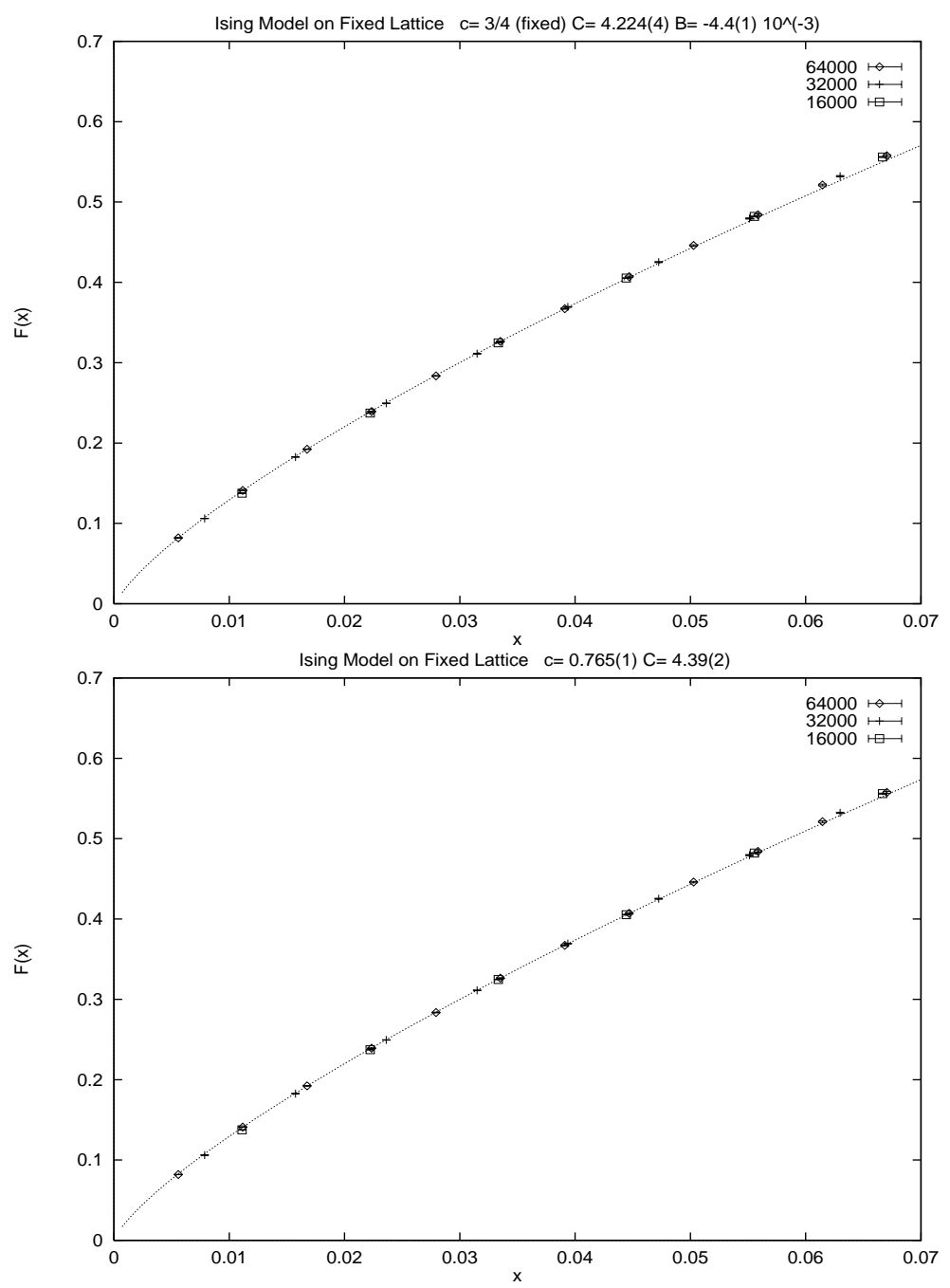

Figure 1: (a) Data for $F_{\phi}^{\text {Flat }}(x)$, as defined in Eq. (24), for small values of $x=\frac{r}{N^{1 / 2}}$ in the case of the Ising model on a fixed triangular lattice with $T^{2}$ topology. The fit shown is to Eq. (25a) for $N_{T}=64000$ triangles. (b) Same as (a) but the fit is to Eq. (25b).

Let us now turn to the main task, the determination of the short distance behavior of the spin-spin correlator for the Ising model and the three-states Potts model coupled to gravity. The Ising model on a regular lattice has a second order transition at a critical temperature $\beta_{c}^{\text {flat }}$ and the corresponding conformal field theory has central charge $c=1 / 2$. The spin field has scaling dimension $\Delta_{0}=1 / 8$ and the dressed scaling dimension after coupling to two-dimensional quantum gravity is $\Delta=1 / 3$. The tree-states Potts model corresponds to a $c=4 / 5$ conformal field theory and the dressed scaling dimension of the spin field in this case is $\Delta=2 / 5$. 

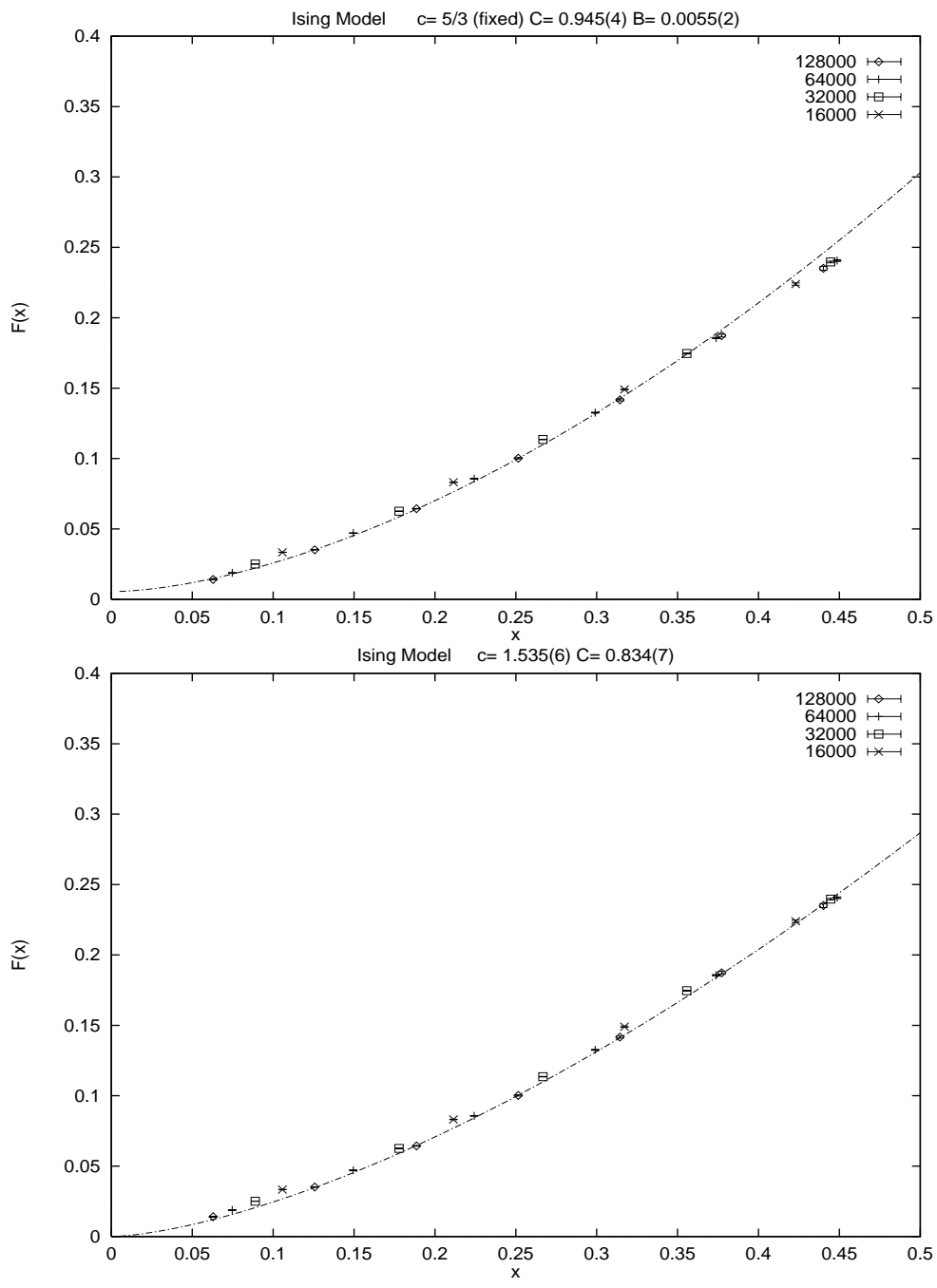

Figure 2: (a) Same as Fig. 1 but for the Ising model coupled to quantum gravity. The fit shown is to Eq. (25a) with exponent $4 / 3$ for $N_{T}=128000$ triangles. (b) Same as $(a)$ but the fit is now to Eq. (25b).

The prediction for short distance scaling is then

$$
F_{\phi}(x) \propto x^{d_{h}(1-\Delta)-1} \quad \text { for } \quad x \ll 1,
$$

i.e. (using the value $d_{h}=4$ )

$$
\begin{aligned}
& F_{\phi}^{I \text { sing }}(x) \propto x^{5 / 3} \quad \text { for } \quad x \ll 1 \\
& F_{\phi}^{\text {Potts }}(x) \propto x^{7 / 5} \text { for } \quad x \ll 1 .
\end{aligned}
$$

In order to calibrate the expected accuracy with which the exponent $d_{h}(1-\Delta)-1$ can be extracted we have performed simulations of an Ising spin system on a flat lattice (with periodic boundary conditions) and measured the known function

$$
F_{\phi}^{\text {Flat }}(x) \propto x^{3 / 4} \quad \text { for } \quad x \ll 1 \text {, }
$$



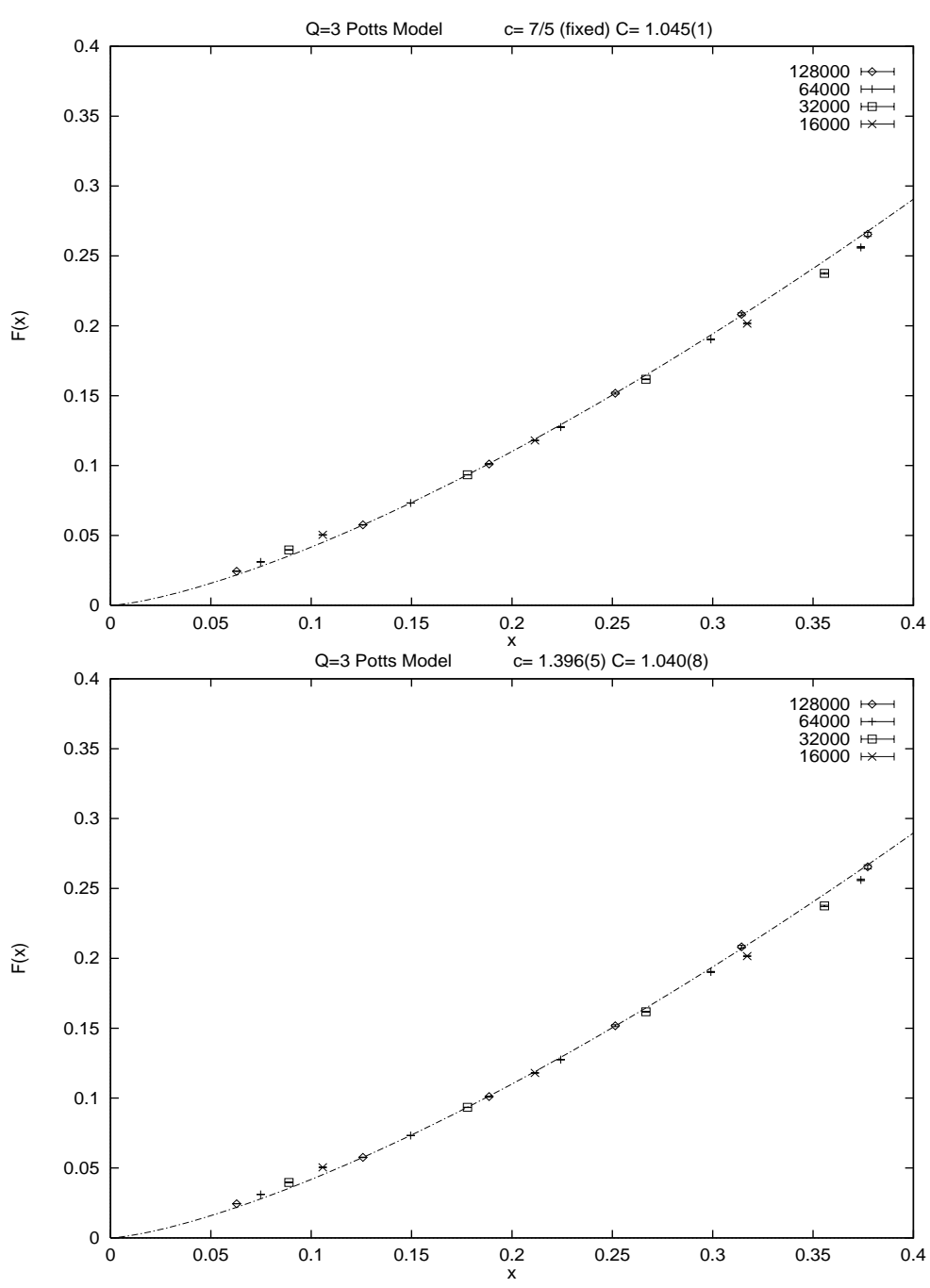

Figure 3: (a) Same as Fig. 1 $1 \mathrm{a}$ in the case of the three-states Potts model coupled to quantum gravity. The fit shown is to Eq. (25a) with exponent $7 / 5$ for $N_{T}=128000$ triangles. (b) Same as in (a) but the fit is to Eq. (25b).

where $x=r / \sqrt{N}$ and $r$ is the distance in lattice units between a spin and its "spherical shell". This rather unusual way of measuring correlation functions works quite well as one can see from the results shown in Table 11. We performed simulations on regular toroidal triangular lattices of sizes 16000-64000 triangles. In order to understand the size of finite size effects and the effect of the function $f(x)$ we show the results of the fits to the following functional forms:

$$
\begin{aligned}
F_{\phi}^{\text {Flat }}(x) & =C x^{3 / 4}+B, \\
& =C x^{c}, \\
& =C x^{c}+B .
\end{aligned}
$$

The constant $B$ in Eqs. (25a) and (25d) reflects the fact that the volume element is discrete with a smallest unit and the spin correlated with itself in the volume element at the shortest distance is 1 . The data is shown in Fig. 1 together with the 
best fit to the largest lattice. It is clear that the determination of the exponent $c$ is in excellent agreement with the theory. It is important that we only use $x \ll 1$ if we fit to Eqs. (25). It is pleasant surprise that the continuum formulae are valid even for small values of $r$. For $x>0.06$ the function $f$ present in Eq. (18) will play an important role.

For the Ising model and the three-states Potts model coupled to gravity we repeat the analysis performed for the regular lattice. We fit the data to Eqs. (25a), with exponents $5 / 3$ and $7 / 5$ respectively, $(25 b)$ and $(25 \mathrm{~g})$ for $x<0.45$. The results are shown in Table 2 for the Ising model and Table 3 for the three-states Potts model. The corresponding plots including the best fit to the largest lattice are shown in Fig. $2 a$ and Fig. $2 b$ for the Ising model and in Fig. 3 $a$ and Fig. $3 b$ for the three-states Potts model.

Our results are consistent with the exponents conjectured in Eqs. (23a) and (23b). We find that simulating the largest lattices, 128000 triangles, is important for obtaining enough data points in the relevant region $x<0.45$. The fits to the predicted behavior Eq. (25a) are good and the finite size correction $B$ approaches zero as the volume increases. Even if the exponent $c$ is allowed to vary, as in the fits to Eqs. (25b) and (25a), it approaches its predicted value convincingly as the volume is increased. The small discrepancy is consistent with finite size effects, which clearly are more important than the statistical errors quoted in Tables 2 and 3. We find that the difference in the values of $c$ obtained from the fits to Eqs. (25b) and $(25 c)$ gives a measure of the systematic errors entering from varying the range and type of the fits.

\begin{tabular}{|c|cc|cc|ccc|}
\hline$N_{T}$ & $C$ & $B$ & $c$ & $C$ & $c$ & $C$ & $B$ \\
\hline \hline 16000 & $4.276(5)$ & $-0.0088(2)$ & $0.7806(8)$ & $4.62(2)$ & $0.754(7)$ & $4.32(7)$ & $-0.008(1)$ \\
32000 & $4.248(4)$ & $-0.0062(2)$ & $0.769(1)$ & $4.44(2)$ & $0.756(4)$ & $4.32(5)$ & $-0.005(1)$ \\
64000 & $4.224(4)$ & $-0.0044(1)$ & $0.765(1)$ & $4.39(2)$ & $0.753(4)$ & $4.25(4)$ & $-0.0038(7)$ \\
\hline
\end{tabular}

Table 1: Results for the fits to Eqs. 25a), (25b) and (25c) for the Ising model on a fixed triangular lattice with $T^{2}$ topology.

\begin{tabular}{|c|cc|cc|ccc|}
\hline$N_{T}$ & $C$ & $B$ & $c$ & $C$ & $c$ & $C$ & $B$ \\
\hline \hline 16000 & $0.880(4)$ & $0.0173(4)$ & $1.432(6)$ & $0.770(6)$ & $1.51(1)$ & $0.799(8)$ & $0.0063(4)$ \\
32000 & $0.923(3)$ & $0.0108(2)$ & $1.470(3)$ & $0.793(3)$ & $1.545(5)$ & $0.829(4)$ & $0.0054(1)$ \\
64000 & $0.940(3)$ & $0.0075(1)$ & $1.497(4)$ & $0.805(4)$ & $1.565(4)$ & $0.841(4)$ & $0.0043(1)$ \\
128000 & $0.945(4)$ & $0.0054(2)$ & $1.535(6)$ & $0.834(7)$ & $1.596(6)$ & $0.870(7)$ & $0.0036(1)$ \\
\hline
\end{tabular}

Table 2: Same as in Table 1 for the Ising model coupled to quantum gravity. 


\begin{tabular}{|c|cc|cc|ccc|}
\hline$N_{T}$ & $C$ & $B$ & $c$ & $C$ & $c$ & $C$ & $B$ \\
\hline \hline 16000 & $0.947(1)$ & $0.0098(1)$ & $1.291(4)$ & $0.880(5)$ & $1.331(8)$ & $0.893(6)$ & $0.0056(6)$ \\
32000 & $0.993(3)$ & $0.0050(3)$ & $1.348(3)$ & $0.959(5)$ & $1.425(7)$ & $1.007(7)$ & $0.0077(3)$ \\
64000 & $1.013(2)$ & $0.0024(2)$ & $1.371(2)$ & $0.991(4)$ & $1.447(5)$ & $1.047(6)$ & $0.0065(2)$ \\
128000 & $1.044(4)$ & $0.0003(3)$ & $1.396(5)$ & $1.040(8)$ & $1.470(8)$ & $1.10(1)$ & $0.0055(3)$ \\
\hline
\end{tabular}

Table 3: Same as in Table 1 for the three-states Potts model coupled to quantum gravity.

\section{Discussion}

We have provided substantial evidence for the conjecture put forward in 18 that the the two-point correlator in a unitary conformal field theory translates the two-point correlator of the same theory coupled to quantum gravity in the following way:

$$
S_{\phi}^{f l a t}(R ; V)=\frac{R}{R^{2 \Delta_{0}}} f^{f l a t}(R / \sqrt{V}) \rightarrow S_{\phi}(R ; V)=\frac{R^{d_{h}-1}}{R^{d_{h} \Delta}} f\left(R / V^{1 / d_{h}}\right),
$$

where $f(0)>0, R$ is the geodesic distance as defined above and $d_{h}$ is the fractal dimension of space-time.

A number of questions still need to be answered before we have a complete understanding of the concept of invariant correlation functions in two-dimensional quantum gravity. First, the value of $d_{h}$ as a function of the central charge $c$ of the conformal field theory should be clarified. Some formal arguments suggest that $d_{h}=2 m$ for a $(m, m+1)$ conformal theory coupled to gravity. This would imply that $d_{h} \rightarrow \infty$ for $c=1-6 / m(m+1) \rightarrow 1$. For the three-states Potts model $m=4$, i.e. the value of $d_{h}=8$. This is definitely not seen in the computer simulations, where particularly the data for $n_{1}(r ; N)$ favor $d_{h}=4$. However, one could argue that the systems considered so far are much too small for observe $d_{h}=8$, since $(128000)^{1 / 8} \approx 4$. While this might be true, it contradicts the fact that finite size scaling works very well for the three-states Potts model coupled to quantum gravity already for considerable smaller systems and we get the correct critical exponents. All the scaling arguments of [17, 18] yield $d_{h}=4$ with quite small error; the only significant discrepancy being that of the height of the maximum of the distribution $n_{\phi}(r ; N)$ for the three-states Potts model. According to Eq. (21) it should scale as $N^{d_{h}(1-\Delta)-1}$, but using the theoretical value $\Delta=7 / 5$ we obtain $d_{h}=4.32(2)$ from collapsing the $64 K-128 K$ configurations. Finite size effects, however, play an important role since the value of $d_{h}$ extracted this way decreases rapidly with system size towards the value 4 . We will report more details in the future [25].

Next, one should understand the tail of the distribution $n_{\phi}(r ; N)$ for $r \gg N^{1 / d_{h}}$. The following simple argument indicates that the distribution should be identical to that of pure gravity: for such large values of $R$ the universes we observe are essentially one-dimensional, since they have to be long tubes of almost no transverse extension. One-dimensional universes cannot (contrary to genuine two-dimensional universes) have non-trivial matter interactions unless they are long range, which is 
not the case here. Consequently there should be no difference between pure gravity and gravity including matter fields. This is only a heuristic argument, but if true it rules out the existence of a single scaling variable $x$ as in Eq. (19) for the whole range of $x$ unless $d_{h}=4$, which is the value for pure gravity. The argument might be too simple since preliminary results for $c=-2$ conformal field theories coupled to gravity indicate both a common scale variable for all values of $x$ and a $d_{h}<4$ [26]. The $c=-2$ matter theory is on the other hand non-unitary and the heuristic arguments might be still be valid for unitary theories.

Finally, it would be most interesting to be able to prove analytically the results reported above and extend the concept of operator product expansion initiated in [15] to the case of unitary conformal field theories coupled to two-dimensional quantum gravity.

\section{References}

[1] V. Knizhnik, A. Polyakov and A. Zamolodchikov, Mod.Phys.Lett A3 (1988) 819.

[2] F. David, Mod.Phys.Lett. A3 (1988) 1651; J. Distler and H. Kawai, Nucl.Phys. B321 (1989) 509.

[3] M. G. Harris and J. Ambjørn, "Correlation Functions in the Multiple Ising Model Coupled to Gravity", NBI-HE-96-04 (hep-th/9602028).

[4] F. David, Nucl.Phys. B368 (1992) 671.

[5] J. Ambjørn, B. Durhuus and J. Fröhlich, Nucl.Phys. B257 (1985) 433; B270 (1986) 457; B275 (1986) 161-184.

[6] J. Ambjørn, B. Durhuus J. Fröhlich and P. Orland, B270 (1986) 457; B275 (1986) 161.

[7] F. David, Nucl.Phys. B257 (1985) 45; Nucl.Phys. B257 (1985) 543.

[8] V.A. Kazakov, I. Kostov and A.A. Migdal, Phys.Lett. B157 (1985) 295; Nucl.Phys. B275 (1986) 641.

[9] J. Ambjørn and Y. Watabiki, Nucl.Phys. B445 (1995) 129.

[10] H. Kawai, N. Kawamoto, T. Mogami and Y. Watabiki Phys.Lett. B306 (1993) 19.

[11] Y. Watabiki, Nucl.Phys. B441 (1995) 119; Progress of Theoretical Physics, Suppl. 114 (1993) 1.

[12] N. Kawamoto, INS-0972, in Nishinomiya 1992 Proceedings, Quantum Gravity (1992) 112. 
[13] N. Ishibashi and H. Kawai, Phys.Lett. B314 (1993) 190; Phys.Lett. B322 (1994) 67.

[14] M. Fukuma. N. Ishibashi, H. Kawai and M. Ninomiya, Nucl.Phys. B427 (1994) 139.

[15] H. Aoki, H. Kawai, J. Nishimura and A. Tsuchiya, "Operator Product Expansion in Two-Dimensional Quantum Gravity", KEK-TH-454 (hep-th/9511117).

[16] J. Ambjørn, B. Durhuus and T. Jonsson, Phys.Lett. B244 (1990) 403; Mod.Phys.Lett. A6 (1991) 1133.

[17] S. Catterall, G. Thorleifsson, M. Bowick and V. John, Phys.Lett. B354(1995) 58.

[18] J. Ambjørn, J. Jurkiewicz and Y. Watabiki, Nucl.Phys. B454 (1995) 313.

[19] C.F. Baillie and D.A. Johnston, Phys.Lett. B286 (1992) 44.

[20] S. Catterall, J. Kogut and R. Renken, Phys.Lett. B292 (1992) 277.

[21] J. Ambjørn, B. Durhuus, T. Jonsson and G. Thorleifsson, Nucl.Phys. B398 (1993) 568.

[22] J. Ambjørn, G. Thorleifsson and M. Wexler, Nucl.Phys. B439 (1995) 187.

[23] D.V. Boulatov and V.A. Kazakov, Phys.Lett. B184 (1987) 247.

[24] J. M. Daul, "Q-states Potts Model on a Random Planar Lattice", LPTENS 94 (hep-th/9502014).

[25] J. Ambjørn, K. Anagnostopoulos, U. Magnea and G. Thorleifsson, to appear.

[26] J. Ambjørn, K. Anagnostopoulos, L. Jensen, N. Kawamoto, K. Yotsuji and Y. Watabiki, to appear. 